
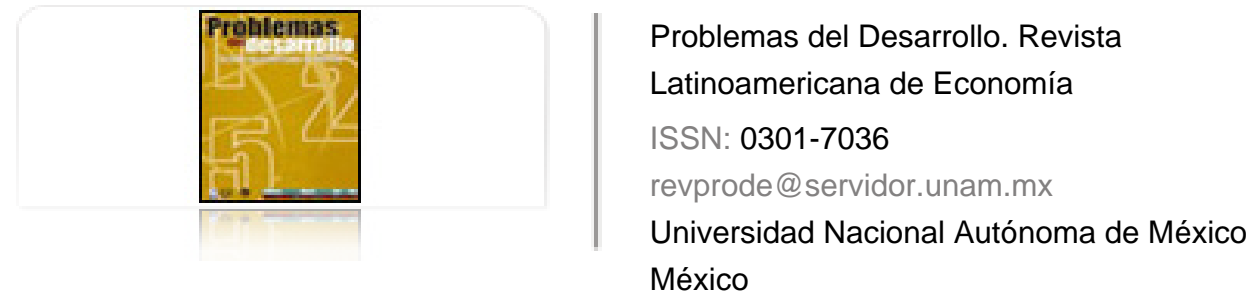

Craviotti, Clara Virginia

Dinámicas territoriales pampeanas y microemprendimientos agrarios en sujetos vulnerables Problemas del Desarrollo. Revista Latinoamericana de Economía, vol. 40, núm. 156, enero-marzo, 2009, pp. 151-172

Universidad Nacional Autónoma de México

Distrito Federal, México

Disponible en: http://www.redalyc.org/articulo.oa?id=11820096003

Cómo citar el artículo

Número completo

- Más información del artículo

Página de la revista en redalyc.org

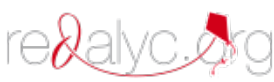

Sistema de Información Científica

Red de Revistas Científicas de América Latina, el Caribe, España y Portugal Proyecto académico sin fines de lucro, desarrollado bajo la iniciativa de acceso abierto 


\title{
DINÁMICAS TERRITORIALES PAMPEANAS Y MICROEMPRENDIMIENTOS AGRARIOS EN SUJETOS VULNERABLES
}

\author{
Clara Virginia Craviotti*
}

Fecha de recepción: 28 de agosto de 2008. Fecha de aceptación: 5 de enero de 2009.

\section{Resumen}

Este trabajo analiza la incidencia de las transformaciones productivas en la región pampeana argentina y, en particular, la provincia de Buenos Aires, en el desarrollo de microemprendimientos agrarios por parte de sujetos sociales vulnerables. La consideración en el interjuego de los factores macro y micro permite extraer conclusiones en relación con ciertos factores que inciden en la viabilidad de dichas iniciativas en los contextos peculiares en que se insertan. Desde otra perspectiva, los procesos de amplio alcance que están influyendo en las áreas estudiadas permiten plantear la limitada capacidad de inclusión del agro pampeano en el contexto de la globalización, en tanto la expulsión de pequeños productores se combina con canales reducidos para posibilitar el ingreso "desde abajo" al sector, como opción ocupacional para quienes experimentan dificultades de inserción en el mercado de trabajo.

Palabras clave: cambios territoriales, empleo agrario, sectores vulnerables, desarrollo local y Argentina.

* Profesora e investigadora de la Maestría de Estudios Sociales Agrarios de FLACSO, Argentina. Correo electrónico: ccraviotti@yahoo.com.

Este artículo se inscribe en el marco del PICT 38175, "La incorporación a la producción agropecuaria de sujetos en situación de vulnerabilidad social. Trayectorias y modalidades de inserción”, financiado por la Agencia Nacional de Promoción Científica y Tecnológica, Argentina. 


\section{Abstract}

This study analyzes the incidence of productive transformations in the Pampas region of Argentina and, in particular, the province of Buenos Aires, in the development of agrarian micro-undertakings by vulnerable social sectors. Looking at the interplay of macro and micro factors makes it possible to come to conclusions about certain factors affecting the viability of these initiatives in the peculiar contexts in which they occur. From another perspective, the broad-ranging processes that are influencing the areas studied make it possible to establish the limited capacity for including Pampas farming in the context of globalization, while the expulsion of small producers combines with limited channels to make possible the entry "from below" into the sector an occupational option for those who experience difficulties entering the labor market.

Key words: territorial changes, farm employment, vulnerable sectors, local development and Argentina.

\section{Résumé}

Ce travail analyse l'incidence des transformations de la production dans la Pampa argentine, notamment la province de Buenos Aires, sur le développement de microentreprises agricoles par des sujets sociaux vulnérables. La prise en compte de l'interaction des facteurs macro y micro permet de tirer des conclusions en rapport avec certains facteurs qui influent sur la viabilité de telles iniciatives dans les contextes particuliers dans lesquels ils s'inscrivent. Si on se place sous un autre angle, les processus de grande portée qui entrent en jeu dans les domaines étudiés permettent d'établir la faible capacité d'inclusion du secteur agricole de la Pampa dans le contexte de la globalisation, tant que l'expulsion de petits producteurs se combine avec l'étroitesse des canaux qui permettent à ceux qui s'affrontent à des difficultés d'insertion sur le marché du travail d'entrer dans le secteur "depuis le bas" et trouver ainsi une option d'activité.

Mots clés: changements territoriaux, emploi agricole, secteurs vulnérables, développement local et Argentine.

\section{Resumo}

Este trabalho analisa a incidência das transformações produtivas na região pampiana argentina e em particular no interior de Buenos Aires, no desenvolvimento de microempreendimentos agrários por parte de sujeitos sociais vulneráveis. A consideração do interjogo dos fatores macro e micro permitem tirar conclusões em relação com certos fatores que incidem na viabilidade dessas iniciativas nos contextos peculiares nos quais se agregam. Desde outra perspectiva, os processos de amplo alcance que estão influindo nas áreas estudadas permitem expor a limitada capacidade de inclusão do agro pampiano no contexto de globalização, enquanto a expulsão de pequenos produtores se combina com canais reduzidos para possibilitar o ingresso "desde zero" ao setor, como opção ocupacional para aqueles que experimentam dificuldades de ingresso ao mercado de trabalho.

Palavras chave: mudanças territoriais, emprego agrário, setores vulneráveis, desenvolvimento local e Argentina.

\section{Desarrollo}




\section{Introducción}

las últimas dos décadas la actividad agropecuaria ha experimentado profundas
transformaciones en Argentina, a partir del interjuego de un conjunto de facto-
res: la influencia del proceso de globalización, que se traduce en una creciente internacionalización de capitales e interpenetración de los mercados, así como en la adopción de estándares de calidad demandados por los mercados internacionales, y los desarrollos tecnológicos que modificaron de manera sustancial las condiciones de producción en la mayoría de las actividades agroalimentarias. Todo ello en el marco de redefiniciones del papel del Estado, que se tradujeron en una menor capacidad de incidir en los procesos económicos y sociales con vistas a revertir las tendencias excluyentes experimentadas por la estructura agraria.

En lo que refiere al mercado de trabajo se advierte la reducción de la población ocupada en la actividad y un mayor énfasis en el trabajo transitorio, junto con transformaciones significativas en la configuración de los mercados de trabajo urbano y rural. La creciente convergencia entre ambos se expresa en fenómenos como la pluriactividad y la creciente residencia urbana de la mano de obra ocupada en actividades agropecuarias, aunque gran parte de ésta aún reside en áreas rurales y en localidades pequeñas, funcionalmente dependientes del sector agropecuario.

El balance de estas tendencias arroja un agro de características concentradas, que no sólo ha expulsado trabajadores, sino también productores, en su mayoría de índole familiar. Paradójicamente, se observa el ingreso de nuevos actores, tanto en actividades que por tradición han constituido la base de inserción del país en los mercados internacionales —el caso de los cereales y oleaginosos—, así como también en varias producciones agroindustriales que en los últimos años han incrementado su inserción exportadora.

Si bien los requerimientos que presentan la mayoría de las actividades agropecuarias implican importantes barreras a la entrada, lo que genera un sesgo en favor de la gran empresa, el panorama de ingreso de nuevos agentes en el sector muestra rasgos de heterogeneidad, ya sea porque incluye la participación de pequeños inversionistas mediante mecanismos de captación de capital —como es el caso de los pools o consorcios de siembra-, o bien porque ciertas actividades — como las producciones no tradicionales orientadas a mercados segmentados- son factibles de ser llevadas a cabo en superficies reducidas, mostrando de hecho el predominio de agentes sin antecedentes previos en la actividad.

Otras situaciones de ingreso al sector son protagonizadas por sujetos sociales vulnerables, que se incorporan a la actividad productiva con la puesta en juego de un 
capital reducido y el trabajo personal o familiar. En este caso, puede plantearse una serie de interrogantes: ¿qué características asume la actividad agropecuaria en estos sujetos? ¿Cuál es su significado dentro de las estrategias familiares de vida? A partir de su situación de vulnerabilidad y su condición de "nuevos" productores, ¿qué mecanismos desarrollan para hacer frente a las barreras de acceso al sector? ¿Hasta qué punto se conectan las actividades desempeñadas con procesos de desarrollo local en las áreas en que se insertan?

En este artículo se analizan algunas de las modalidades que se asumen con este tipo de actividades, a veces denominadas "microemprendimientos", a partir de las trayectorias ocupacionales previas de los agentes sociales que las encaran, así como ciertas características de estos sujetos en tanto agentes productivos agrarios. Asimismo, y teniendo en cuenta que las estrategias asumidas por los actores se interceptan con los cambios producidos en las tramas productivas locales, se consideran ciertos elementos contextuales que inciden en el surgimiento y en la viabilidad de sus emprendimientos, tomando la última década como periodo fundamental de estudio. La metodología incluye el análisis de datos secundarios, combinados con técnicas de análisis cualitativas, con base en entrevistas semiestructuradas a informantes calificados y casos seleccionados. El trabajo de campo en que se apoya este artículo ha sido efectuado en cuatro partidos de la provincia de Buenos Aires, pertenecientes a la región pampeana argentina. Se trata de ámbitos locales caracterizados por una baja densidad poblacional, importancia de las actividades agropecuarias dentro de la estructura del empleo, y relativa distancia a ciudades grandes o intermedias. ${ }^{1}$

\section{Los cambios en el agro pampeano}

El agotamiento del modelo de acumulación basado en la industrialización sustitutiva de importaciones, que se presenta hacia 1970 tanto en Argentina como en otros países periféricos, dio lugar a un nuevo modelo que alcanza su máxima expresión en los años noventa, con las medidas de política entonces adoptadas: la determinación

1 Si se toma el criterio de ruralidad considerado por el Instituto Nacional de Estadística y Censos (INDEC) de Argentina, algunas localidades de los partidos estudiados serían clasificadas como urbanas, por superar los 2,000 habitantes; si se consideran criterios de ruralidad ampliada (de Ferranti y otros, 2005) con base en la Organización de Cooperación para el Desarrollo Económico (OCDE) —es decir, una densidad de población menor a los 150 habitantes por kilómetro cuadrado y una distancia a centros urbanos de más de 100,000 habitantes superior a la hora de viaje — todas, excepto una de las analizadas, serían definidas como rurales. 
de un tipo de cambio fijo y convertible, la privatización de empresas estatales, la desregulación de los mercados y la apertura externa (Rofman, 2000). En el caso del agro, se disolvieron diversos organismos estatales de regulación y se liberaron los cupos de siembra, cosecha, elaboración y comercialización de diversas producciones. ${ }^{2}$ Las medidas de desregulación impositiva eliminaron los impuestos y tasas sobre las exportaciones, rebajaron los aranceles a la importación de insumos y de productos agropecuarios y suprimieron diversos impuestos a distintas operaciones comerciales (Barky y Fernández, 2005).

En este contexto de profunda redefinición de las reglas de juego, algunos autores consideraron que el sector agrario fue de los más dinámicos de la economía, en tanto la existencia de un marco macroeconómico de estabilidad y la apertura de los mercados indujeron a la adopción de diversas tecnologías biológicas y químicas y a la modernización de los sistemas productivos (Chudnovsky et al., 1999). Destacan la incorporación de la siembra directa, en particular de la soja transgénica, y los aumentos significativos en la compra de maquinaria e insumos clave como los fertilizantes. Sin embargo, la propia naturaleza del modelo adoptado necesariamente tendía a la concentración del poder económico y al aumento de escala de las empresas (Lattuada, 2000), circunstancia que quedó reflejada en la disminución de la cantidad de explotaciones (de 21\% para el periodo intercensal 1988-2002) y en el aumento de la superficie promedio, que pasó de 469 a 588 hectáreas. Las políticas aplicadas repercutieron de manera desigual en las diferentes regiones, en función de sus condiciones institucionales, el tipo de producciones predominantes y los agentes participantes. Así en la región pampeana el proceso de concentración fue más agudo: las explotaciones agropecuarias disminuyeron en $29 \%$ y en lo particular las de menor dimensión fueron las más afectadas.

La modificación del marco macroeconómico a partir de 2002 generó condiciones para las actividades agropecuarias diferentes de las imperantes en la década previa, a las que se aúna un contexto internacional signado por una mayor demanda de materias primas. Por una parte, la marcada devaluación de la moneda argentina significó

2 Las medidas vinculadas con la desregulación de los mercados se tradujeron en la disolución de la Junta Nacional de Granos, la Junta Nacional de Carnes, la Corporación Argentina de Productores de Carnes, la Dirección Nacional del Azúcar, el Mercado Consignatario Nacional de Yerba Mate, la Comisión Reguladora de la Producción y Comercio de Yerba Mate, el Instituto Forestal Nacional y el Mercado de Concentración Pesquera. También se liberaron los cupos de siembra, cosecha, elaboración y comercialización de caña de azúcar, yerba mate y viñedos, uva y vino. Se eliminaron las regulaciones del mercado de leche e industria láctea (Barsky y Fernández, 2005). 
una gran transferencia de recursos hacia las actividades vinculadas a la exportación, parcialmente compensada con las retenciones (impuestos) a las exportaciones y por aumentos en los insumos y salarios. La recuperación de la recesión iniciada en 1998 y la creciente expansión del mercado interno generaron procesos expansivos que repercutieron en la demanda de los productos agropecuarios. Por otra parte, la pesificación de las deudas bancarias de los productores agropecuarios evitó la pérdida de unidades fuertemente endeudadas, como producto de la ecuación económica desfavorable durante la segunda mitad de la década de los años noventa (Barsky y Fernández, 2005). También han cambiado las orientaciones en materia del papel del Estado, procurando regular los precios internos de algunos alimentos y subsidiar los costos de ciertos insumos, aunque gran parte de las condiciones heredadas de la década previa no se han modificado.

En el caso de la dinámica región pampeana, proveedora de casi $80 \%$ de las exportaciones agrícolas en 2004 (Banco Mundial, 2006), la profundización del proceso de modernización iniciado en los setenta ha dado como resultado una producción agrícola sustentada mayoritariamente en cuatro cultivos (soja, maíz, trigo y girasol) y el desplazamiento de la actividad ganadera. Como lo indican los datos cuantitativos disponibles, se trata de tendencias de largo alcance, que se han visto acentuadas con la modificación de la paridad cambiaria en 2002. En la provincia de Buenos Aires, corazón de la región pampeana, el crecimiento de la proporción de la superficie implantada con soja se acompaña de un reforzamiento de los cuatro cultivos, en lo referente a su participación en el total de superficie implantada (véase el cuadro 1).

Además del factor económico y la ausencia de políticas que favorecieran la diversificación productiva, impulsaron el desarrollo de semillas adaptadas a diferentes zonas en la dirección indicada y actuaron en la modificación del régimen de lluvias. Así es como en áreas tradicionalmente ganaderas, como la del noreste y centro-oeste de la provincia, donde la soja en la práctica no existía, ahora abarca superficies importantes. Debido a que se carecen de datos recientes de superficie implantada que cubran la totalidad de las producciones, no es posible concluir si este incremento se dio por la siembra en campos antes no cultivados o a costa de desplazar otros cultivos (inclusive forrajeros). No obstante, los informantes consultados indican que en el caso de la ganadería bovina el desplazamiento afecta fundamentalmente a producciones que requieren de pasturas cultivadas, como la invernada o engorde y, en segundo lugar, a la actividad tambera: "El tambero resiste por cultura, porque viene de generación en generación [...] Les cuesta más desprenderse y algunos la siguen luchando, el invernador levantó los boyeros y sembró. Porque es mucho más dinámico, cambiante, son 
Cuadro 1

Provincia de Buenos Aires. Superficie implantada total y con los principales cultivos de cosecha fina y gruesa (2001/2-2005/6), según zonas agroproductivas, en porcentajes*

\begin{tabular}{|c|c|c|c|c|c|c|c|c|c|}
\hline \multirow[b]{2}{*}{ Zonificación } & \multirow{2}{*}{$\begin{array}{l}\text { Superficie implantada } \\
\text { total } 1^{0} \text { y } 2^{\circ} \\
\text { ocupación }(2001 / 2)\end{array}$} & \multicolumn{2}{|c|}{ Maíz } & \multicolumn{2}{|c|}{ Soja } & \multicolumn{2}{|c|}{ Trigo } & \multicolumn{2}{|c|}{ Girasol } \\
\hline & & $2001 / 2$ & $2005 / 6$ & $2001 / 2$ & $2005 / 6$ & $2001 / 2$ & $2005 / 6$ & $2001 / 2$ & $2005 / 6$ \\
\hline $\begin{array}{l}\text { 1. Agrícola-ganadera } \\
\text { del norte }\end{array}$ & $2,201,057$ & 12 & 10 & 54 & 63 & 17 & 11 & 0 & 1 \\
\hline $\begin{array}{l}\text { 2. Ganadero-agrícola } \\
\text { del centro norte }\end{array}$ & 336,565 & 9 & 8 & 33 & 36 & 14 & 8 & 2 & 2 \\
\hline $\begin{array}{l}\text { 3. Ganadera del } \\
\text { noreste }\end{array}$ & 120,481 & 8 & 11 & 14 & 29 & 11 & 11 & 2 & 1 \\
\hline $\begin{array}{l}\text { 4. Ganadera del } \\
\text { centro este }\end{array}$ & $1,432,706$ & 9 & 7 & 18 & 22 & 16 & 13 & 5 & 4 \\
\hline $\begin{array}{l}\text { 5. Ganadero-agrícola } \\
\text { del oeste }\end{array}$ & 399,916 & 10 & 16 & 17 & 29 & 9 & 4 & 12 & 10 \\
\hline $\begin{array}{l}\text { 6. Ganadera del } \\
\text { centro oeste }\end{array}$ & 984,874 & 10 & 12 & 23 & 45 & 14 & 9 & 6 & 7 \\
\hline 7. Mixta del sur & $5,408,213$ & 3 & 3 & 5 & 13 & 34 & 41 & 13 & 17 \\
\hline $\begin{array}{l}\text { 8. Mixta compleja } \\
\text { del noroeste }\end{array}$ & $1,194,663$ & 11 & 10 & 37 & 48 & 15 & 9 & 2 & 2 \\
\hline Total & $12,078,474$ & 7 & 7 & 21 & 31 & 23 & 24 & 8 & 9 \\
\hline
\end{tabular}

Fuente: Censo Nacional Agropecuario de 2002; Ministerio de Asuntos Agrarios de la provincia de Buenos Aires (datos de la campaña 2005/06).

Nota: Los partidos fueron agrupados según la zonificación propuesta por Andrés Barsky (1997).Los datos referidos a la campaña 2005/6 deben ser tomados con recaudos, por tratarse de estimaciones.

* Los datos censales y de superficie implantada fueron reprocesados con la colaboración de Alejandro Gerardi.

ciclos más cortos. En cambio, cargarse de genética para tener un rodeo o [...] tambo exige otra cosa" (director de escuela agraria, 2007).

Otro de los impactos generados a partir del dinamismo de la soja es el desplazamiento territorial de productores de áreas agrícolas hacia áreas ganaderas, donde arriendan campos para ubicar su hacienda. El incremento del valor de la tierra ha distorsionado un mercado en el que el valor del arrendamiento históricamente se fijaba en función del rendimiento que se obtiene en kilos de novillo, lo que alienta conductas rentísticas y afecta la permanencia de los productores de menor tamaño.

"Toda la gente de Tandil arrendó el campo para sembrar y la gente que tenía vacas se vino para este lado, para la zona más marginal. ¿Esto que logró? Que todos los pedacitos de campo que se pagaban a 65 kilos llegaron a pagar 120 kilos la hectárea. Porque a esta persona de Tandil no le importaba el ternero, lo que le importaba era no quedarse sin vacas, entonces invertía el total del ternero, porque cuando se va a vender pesa 240-250 kilos y cuando lo trajo para acá estaba pagando la mitad, 120. Es la mitad descontando todos los gastos, perdía plata, pero qué le importaba perder acá, si allá ganaba dos veces más, entonces eso trajo aparejado que acá empezaran a 
subir los precios; gente que toda la vida fue ganadera tuvo que salir a arrendar campo mucho más caro" (técnico apícola, 2007).

Entre las modificaciones que tiene la ganadería empujada por la agriculturización que ha habido, hay muchos más campos arrendados. [...] los alquileres son muy altos, entonces para un ganadero poco eficiente que quizás esté en los últimos años de su vida productiva y no tenga un hijo, o un familiar muy cercano que quiera continuar, está muy tentado a alquilar por los altos valores. Entonces, las vacas las tenemos, pero los propietarios de las vacas son cada vez menos (funcionario de Secretaría de Producción y Desarrollo Rural del municipio, 2007).

Este proceso no es ajeno a la consolidación de formas de organización de la producción más deslocalizadas (como los pools o consorcios de siembra), que se apoyan, aunque no exclusivamente, en capitales externos al agro. El uso más intensivo de insumos y paquetes tecnológicos ha elevado las exigencias de capital volcado a la agricultura, favoreciendo estos esquemas, de características más concentradas (Gorenstein, 2000; Gutman y Gorenstein, 2003).

Las repercusiones sobre el empleo rural del proceso de agriculturización y concentración productiva son importantes. Por un lado, en la región pampeana y, en particular, en la provincia de Buenos Aires, la medición censal de 2001 registró una importante caída de la población ocupada en actividades agropecuarias con respecto a la de 1991, tanto en términos absolutos como relativos. El comportamiento por zona ha sido dispar, acentuándose su disminución en varias de las zonas ganaderas (véase cuadro 2).

Cuadro 2

Provincia de Buenos Aires. Población ocupada en la rama agraria (1991-2001), según zonas agroproductivas, en números absolutos y porcentajes

\begin{tabular}{lcccccc}
\hline & \multicolumn{7}{c}{ 1991 } & \multicolumn{7}{c}{ 2001 } \\
\cline { 2 - 6 } Zonificación & $\begin{array}{c}\text { Total de } \\
\text { ocupados }\end{array}$ & $\begin{array}{c}\text { Total ocupados en } \\
\text { rama agraria }\end{array}$ & $\%$ & Total de ocupados & $\begin{array}{c}\text { Total de } \\
\text { ocupados en } \\
\text { rama agraria }\end{array}$ & $\%$ \\
\hline 1. Agrícola-ganadera del norte & 323,086 & 46,731 & 14 & 272,926 & 29,293 & 11 \\
2. Ganadero-agrícola del centro norte & 204,401 & 18,754 & 9 & 212,178 & 13,252 & 6 \\
3. Ganadera del noreste & 302,626 & 18,985 & 6 & 292,120 & 13,718 & 5 \\
4. Ganadera del centro-este & 190,549 & 38,717 & 20 & 163,848 & 24,910 & 15 \\
5. Ganadero-agrícola del oeste & 12,608 & 3,834 & 30 & 11,132 & 2,632 & 24 \\
6. Ganadera del centro-oeste & 62,656 & 16,119 & 26 & 52,649 & 10,780 & 20 \\
7. Mixta del sur & 588,705 & 76,130 & 13 & 500,209 & 44,030 & 9 \\
8. Mixta compleja del noroeste & 71,765 & 19,840 & 28 & 60,480 & 12,508 & 21 \\
Total & $1,756,396$ & 239,110 & 14 & $1,565,542$ & 151,123 & 10 \\
\hline
\end{tabular}

Fuente: Censo Nacional de Población, Hogares y Vivienda 1991 y 2001, reprocesamientos especiales. 
Si bien se carece de datos censales más actuales sobre población ocupada por ramas de actividad, es posible pensar que esta tendencia no sólo no se ha revertido, sino que en todo caso se ha mantenido o incluso profundizado en los últimos años.

Estos datos deben entenderse a la luz del importante proceso de cambio tecnológico experimentado por las principales actividades agrícolas en que se basa la región. En el caso de la soja, la gran mayoría de la superficie es plantada con siembra directa, tecnología que demanda $30 \%$ menos de mano de obra que la siembra convencional, al eliminar algunas tareas (Benencia y Quaranta, 2007).

El carácter fuertemente mecanizado de la agricultura pampeana ha incrementado la transitoriedad del empleo, sólo en parte neutralizado por el crecimiento de las empresas de servicios de maquinaria, que brindan una ocupación más continua a su personal (ya que al tiempo dedicado a las actividades de campo hay que sumarle el requerido por la reparación de los equipos).

Las entrevistas realizadas son coincidentes en la reducción de la mano de obra directa empleada en agricultura, aunque destacan el crecimiento de la ocupación en servicios vinculados, en particular a partir del cambio de la política macroeconómica en 2002, y el consiguiente reposicionamiento de las actividades orientadas a la exportación. No obstante, también es pertinente tener en cuenta que en el caso de los granos la concentración de la comercialización y la integración de funciones en las grandes empresas han contribuido a desarticular actividades previamente realizadas en las localidades.

La existencia de modalidades productivas — como los pools de siembra— refuerza estos procesos, al sustituir algunas funciones de los centros de servicios agrarios, o al desplazar sus demandas hacia ámbitos más lejanos (Gorenstein, 2000).

En relación con la población ocupada en actividades ganaderas, es difícil evaluar la magnitud de los cambios, dada la existencia de tendencias contrapuestas.

La incorporación de tecnología, aunque desigualmente, se expresó en mejoras en el manejo de la alimentación y la reproducción del rodeo (Neiman y otros, 2006; Benencia y Quaranta, 2007). Este hecho puede haber tenido impactos positivos sobre el empleo, al incorporarse tareas más demandantes de trabajo, a pesar de haber disminuido la superficie ganadera.

No obstante, también en este caso se insiste desde ámbitos locales en que hay una tendencia a llevar a cabo más tareas con el mismo personal o quizá menos. Además el proceso de agriculturización no sólo ha incidido sobre el empleo directo en ganadería, sino también en las actividades de servicios vinculadas a esta producción: "La sojización ha hecho un desastre [...] al cerrarse los tambos, que es lo que da más tra- 
bajo en los campos, porque no sólo está el tambero sino el que lleva el alimento para los animales, está el que va a buscar la leche, el camionero, los talleres que arreglan los vehículos que se utilizan en el campo, hay mucha gente que trabaja alrededor de eso" (funcionaria de Acción Social de municipio, 2007).

Para dimensionar estos procesos, es pertinente tener en cuenta que en los habitantes rurales de la provincia de Buenos Aires la ocupación en el sector agropecuario —en particular como trabajador asalariado- era predominante en 2001, más aún en aquellas áreas basadas en la actividad ganadera (véase el cuadro 3).

Cuadro 3

Provincia de Buenos Aires. Población rural ocupada por rama de actividad, según zonas agroproductivas (2001), en porcentajes

\begin{tabular}{lcccc}
\hline & \multicolumn{3}{c}{ Ramas de actividad } \\
Zonificación & Primaria & Secundarias & Terciaria & Total \\
\hline 1. Agrícola-ganadera del norte & 45 & 14 & 41 & 100 \\
2. Ganadero-agrícola del centro-norte & 38 & 15 & 47 & 100 \\
3. Ganadera del noreste & 47 & 11 & 41 & 100 \\
4. Ganadera del centro-este & 55 & 8 & 37 & 100 \\
5. Ganadero-agrícola del oeste & 52 & 6 & 42 & 100 \\
6. Ganadera del centro-0este & 58 & 6 & 36 & 100 \\
7. Mixta del sur & 50 & 10 & 40 & 100 \\
8. Mixta compleja del noroeste & 48 & 11 & 41 & 100 \\
Total provincia & 49 & 11 & 40 & 100 \\
\hline
\end{tabular}

Fuente: Censo Nacional de Población y Viviendas 2001, reprocesamientos especiales. La rama primaria reúne $0.2 \%$ de población ocupada en actividades no incluidas dentro de agricultura, ganadería, caza y silvicultura.

La fuerte expulsión de mano de obra permanente que se produjo en las explotaciones agropecuarias pampeanas formó en las localidades próximas a las zonas productivas una oferta de trabajadores, quienes se insertan en diferentes ocupaciones de características inestables y que no requieren de "credenciales" educativas: el agro, la construcción, el servicio doméstico (en el caso de las mujeres) y changas de diversa índole, ya sea bajo una modalidad combinada o intermitente.

En algunos casos desarrollan actividades productivas en pequeña escala ante las dificultades experimentadas en el mercado de trabajo, en un contexto en que opciones de esta índole fueron promovidas desde diversos ámbitos locales, con recursos en buena medida provenientes de programas nacionales. 


\section{Los microemprendimientos agrarios como opción ocupacional}

A partir de estos elementos, la investigación apuntó a analizar el carácter asumido por las iniciativas de producción agropecuaria encaradas por sujetos vulnerables pertenecientes a áreas rurales y pequeñas localidades pampeanas, ya sea apoyados desde diversos programas o bien desarrollados de forma autónoma. Éstos debían estar orientados al mercado, con base en el control de un capital relativamente reducido, y el trabajo propio o familiar debía tener centralidad para el desarrollo del proceso productivo. ${ }^{3}$ En el ámbito empírico se trabajó en cuatro partidos de la provincia de Buenos Aires y se procuró, en la medida de lo posible, diversificar los casos bajo análisis en función del tipo de producciones encaradas, así como considerar los diferentes contextos socioproductivos e institucionales que condicionaban su desarrollo. ${ }^{4}$

Se tomó la ocupación desempeñada por los sujetos con anterioridad, "al inicio del emprendimiento", como criterio fundamental de vulnerabilidad. Más precisamente, la precariedad de la misma (dada por la carencia de estabilidad o de beneficios sociales) o su carácter no calificado, que se asocia con la percepción de ingresos bajos. No obstante, tendemos a coincidir con Lomnitz (1975) en que a pesar de la precariedad ocupacional, cuando los ingresos son predecibles, brindan otro marco de contención a los sujetos, lo que se traslada al horizonte de su acción y al tipo de iniciativas desarrolladas. En consecuencia, se puede pensar en un "gradiente" de situaciones de vulnerabilidad.

Por otra parte, en las entrevistas se identificaron otros indicadores - que vinieron a corroborar el carácter multidimensional del concepto (Pizarro, 2001)— así como la validez de la selección efectuada. Nos referimos a la temprana edad en que se da

3 Asimismo, se consideró que los casos debían haberse iniciado como productores a partir de 2000 y presentar un mínimo de permanencia en la actividad — definido operativamente como haber comercializado su producción durante al menos una campaña.

4 Las entrevistas a los casos fueron complementadas con otras efectuadas a una diversidad de informantes clave en los ámbitos nacional y local, con entrevistas a casos fronterizos, que por su condición se los consideró "fuera de perfil", pero permitían ilustrar criterios de diferenciación de los emprendimientos, y también a casos negativos, que abandonaron la actividad oportunamente iniciada. Los casos fueron seleccionados a partir de un "banco" de casos potenciales, relevados a partir de la consulta con diversos programas nacionales, organizaciones no gubernamentales, ámbitos municipales locales y referentes locales de organismos de asistencia técnica agropecuaria. Se entrevistaron apicultores, cunicultores, criadores de pollos parrilleros, productores hortícolas, entre otros. Las entrevistas partían de una guía exhaustiva complementada con preguntas espontáneas en función de las respuestas que iban surgiendo, y fueron grabadas en su totalidad. 
la incorporación al trabajo de los sujetos, la presencia en el hogar de menores que trabajan; en el caso de las mujeres, la edad en que tuvieron a su primer hijo; el acceso a la vivienda con casas prestadas o alquiladas, o bien a partir de planes de vivienda subsidiados; el hecho de que sus casas estén sin terminar, o existan situaciones de hacinamiento. Asimismo, el lugar de residencia (en la periferia de las localidades) suele estar asociado a la distancia social con respecto a los estratos mejor ubicados.

La presencia de varios de estos indicadores de forma simultánea nos habla de situaciones de "acumulación de desventajas" (Paugam, 1995, citado por Saraví, 2006). En contrapartida, otros rasgos permiten pensar en (posibles) criterios de diferenciación que se suman a la ya mencionada estabilidad ocupacional y de ingresos. Por ejemplo, el haber podido completar estudios secundarios o haber tenido la posibilidad de capacitaciones no formales; los vínculos desarrollados que posibilitan cierto acceso a recursos; la trayectoria laboral en diversos sectores y ámbitos de actividad, no exclusivamente locales, que dan lugar a experiencias y saberes más amplios.

Un aspecto que surge del análisis es que el grado de vulnerabilidad se vincula con el grado de autonomía de los sujetos que encaran los emprendimientos. Más específicamente, con el papel desempeñado por las instancias institucionales de apoyo —de acompañamiento, supervisión o eventualmente, dirección del proceso- - Si bien en este último caso pueden surgir dudas acerca del carácter "por cuenta propia" de los emprendimientos involucrados, de todas maneras se estimó pertinente su consideración para conocer las repercusiones de este vínculo sobre su desempeño.

En los casos analizados, y dejando de lado a quienes estaban desocupados al inicio de los emprendimientos, las ocupaciones previas fueron agropecuarias en uno de cada cuatro entrevistados (véase el cuadro 4). Casi la totalidad posee algún antecedente personal o familiar en el sector, aunque, por lo general, no como productores directos, sino como asalariados, situación en la que aprendieron el oficio y tuvieron acceso a ciertos recursos productivos como forma de pago parcial de su trabajo. $\mathrm{O}$ bien, la familia disponía de un pequeño terreno donde tenía una huerta o animales de granja. Es clara la reducida experiencia en el desarrollo de actividades que de forma autónoma pueda ser transferible a la nueva labor. Por otra parte, la importancia actual del trabajo asalariado en estos sujetos puede ser considerada como un indicador indirecto de que el emprendimiento no se ha logrado consolidar como opción generadora de ingresos.

En poco más de la mitad de los casos analizados se da la participación de otros miembros familiares en tareas ligadas al emprendimiento. Si se toma la definición de producción familiar aportada por Djurfeldt (Román y Tort, 2005), que hace hincapié 
Cuadro 4

Actividades desarrolladas con anterioridad al inicio del emprendimiento y en la actualidad

\begin{tabular}{|c|c|c|c|c|c|c|c|}
\hline \multirow[t]{2}{*}{ Actividad actual } & \multicolumn{7}{|c|}{ Actividad previa } \\
\hline & $\begin{array}{c}\text { Por cuenta } \\
\text { propia no } \\
\text { agraria }\end{array}$ & $\begin{array}{c}\text { Por cuenta } \\
\text { propia agraria }\end{array}$ & $\begin{array}{l}\text { Asalariada } \\
\text { no agraria }\end{array}$ & Asalariada agraria & Combinación & Desocupado & Total \\
\hline $\begin{array}{l}\text { Por cuenta propia no } \\
\text { agraria } \\
\text { Por cuenta propia } \\
\text { agraria }\end{array}$ & 2 & & & & & 1 & 3 \\
\hline Asalariada no agraria & & & 2 & & & 3 & 5 \\
\hline $\begin{array}{l}\text { Asalariada agraria } \\
\text { Combinación }\end{array}$ & & & 1 & $\begin{array}{l}2 \\
1\end{array}$ & 1 & 1 & $\begin{array}{l}4 \\
4\end{array}$ \\
\hline $\begin{array}{l}\text { Sin otra ocupación/ } \\
\text { ocupaciones esporádicas }\end{array}$ & & & 1 & 2 & & 2 & 5 \\
\hline Total & 2 & 0 & 5 & 5 & 1 & 8 & 21 \\
\hline
\end{tabular}

Fuente: Elaboración propia con base en las entrevistas realizadas. No incluye el trabajo en el emprendimiento productivo.

en los aspectos que posibilitan la autonomía de la unidad - es decir, sobre todo el empleo de mano de obra de la familia y la reproducción de ésta y de la explotación por medio del ingreso predial—, se pone en evidencia la dificultad de los casos analizados para cumplir el segundo criterio, aunque se reconoce que el tiempo transcurrido desde su inicio como productores resulta relativamente escaso para evaluar su grado de consolidación en la actividad. ${ }^{5}$ Sin embargo, cabe resaltar que está presente un elemento característico de estas unidades: la fusión de la economía doméstica con la de la empresa y una forma de cálculo que sólo incluye como costos los gastos monetarios, por lo general no contabilizados.

El tipo de actividad productiva elegida obedece a un conjunto de factores, algunos de los cuales se pueden caracterizar como "circunstanciales" — como la existencia de un apoyo específico para la actividad en los ámbitos local, provincial o nacional一, otros, en cambio, se relacionan con las restricciones particulares que enfrentan estos sujetos. Por ello, no es de extrañar el predominio de la apicultura, en tanto actividad que no requiere de tierra - aunque sí el acuerdo de los dueños de los campos donde se ubicarán las colmenas (véase el cuadro 5).

Tanto los apicultores como los cunicultores se distinguen del resto de los casos estudiados por incorporar capital propio al inicio del emprendimiento, más allá de los apoyos obtenidos de programas o logrados a partir de su participación en redes sociales.

5 La mayoría se inició en los años 2003-2004, lo que se vincula con apoyos recibidos del Programa Nacional de Desarrollo Local y Economía Social "Manos a la Obra". 


\section{ClARA VIRGINIA CRAVIOTTI}

Cuadro 5

Actividades productivas desarrolladas según la intensidad del trabajo demandado

\begin{tabular}{lcc}
\hline Baja intensidad & Mediana intensidad & Alta intensidad \\
\hline Apicultura (10) & Cunicultura (3) & \\
Vivero forestal (1) & Cría de pollos (3) & Horticultura (3) \\
& Cría de chinchillas (1) & \\
\hline
\end{tabular}

Fuente: Elaboración propia con base en las entrevistas realizadas.

Esta situación confirmaría lo señalado por los informantes clave consultados, en el sentido de que en la actualidad la apicultura, si bien es una actividad susceptible de desarrollarse en baja escala, no es del todo apta "para los más pobres".

- [...] Cuando uno piensa en un apicultor, es una tarea más compleja. Quien decide iniciarse ya de entrada sabe que requiere más capital, que requiere más conexiones, que requiere más vida social.

— ¿Por qué?

-Por ejemplo, si a usted se le ocurriera formar un apiario, usted tiene que convencerme a mí, propietario del campo, porque yo le tengo que brindar el campo [...].

- Sí, el tema de la vida social está claro, pero usted dice que tienen más medios económicos, muchos empezaron con 20 colmenas.

—Sí, sí. Pero para ir a verlas se requiere vehículo, o que vayan en remís a verlas [...] Se requiere una camioneta para llevar la salsa, para extraer la miel (funcionario de la Secretaría de producción y desarrollo rural de municipio, 2007).

- [La apicultura] es excelente como un segundo ingreso, siempre y cuando el productor tenga muy claro lo que hace.

- Aun como segundo ingreso, es difícil.

-Y es difícil, porque tiene un costo alto (técnico apícola, 2007).

Por otra parte, las restricciones en materia de capital significan que en el mejor de los casos, este "nuevo" productor va creciendo lentamente en su actividad, con oscilaciones marcadas en el transcurso de su trayectoria. Las limitaciones en recursos productivos condicionan también la magnitud de los ingresos generados y da lugar a que el trabajo en el emprendimiento ocupe el segundo o tercer lugar respecto a otras ocupaciones y, por consiguiente, en los ingresos familiares. Sólo en algún caso es el principal ingreso familiar.

El propio proceso de crecer en la actividad es visto como una mayor exposición al riesgo, si implica descuidar otras actividades. En este sentido, puede decirse que es constante la comparación con otras opciones y ofertas laborales (aunque éstas sean changas, constituyen el "costo de oportunidad" de estos sujetos) y ello tiene su razón de ser en sus trayectorias ocupacionales y en los ingresos reducidos que perciben. 
Estas consideraciones no significan que el emprendimiento no sea valorado: brinda ingresos complementarios (especialmente en etapas del ciclo familiar donde los hijos generan más gastos vinculados al estudio); en el caso de las mujeres, genera un ingreso sobre el que tienen mayor control, que con frecuencia es destinado a mejorar las condiciones de hábitat familiar o el trabajo doméstico; además es un trabajo que si es realizado en el lugar donde residen, les permite estar más cerca de sus hijos. A futuro, el emprendimiento puede ser visto como un posible ahorro (sobre todo en el caso de la apicultura) y dependiendo de la actividad realizada, como una opción de trabajo que implica menos esfuerzo que otras ocupaciones desempeñadas.

La multiocupación de estos sujetos hace que los requerimientos del emprendimiento, e incluso las actividades de capacitación no formal, muchas veces sean pospuestos frente a los planteados por las demás actividades, y se aprovechan los ratos libres los "fines de semana". Ello afecta menos a la apicultura que a otras actividades más intensivas como la cunicultura, donde el menor involucramiento del productor influye en la alimentación y la sanidad de los animales. Cuando el emprendimiento está inserto en redes institucionales, para garantizar el normal desarrollo de las tareas, técnicos o funcionarios municipales han debido diseñar dispositivos (como un fondo de reserva) para asegurar cierta continuidad en los ingresos obtenidos por los productores y también para hacer frente a los gastos demandados por los insumos iniciales de cada ciclo productivo.

Estas apreciaciones nos remiten a los requerimientos específicos de la actividad agropecuaria, que suponen importantes barreras al acceso e implican dificultades adicionales a las que de por sí puede tener cualquier emprendimiento encarado de forma autónoma. Estos aspectos se potencian en el caso de sujetos vulnerables: nos referimos a la incidencia de factores climáticos; a los requisitos bromatológicos que se deben cumplir para comercializar alimentos; al tiempo que transcurre entre el inicio del emprendimiento y la obtención de ingresos; a la estacionalidad de los mismos; a la necesidad de contar con algún mecanismo de acceso a la tierra. La comercialización es otro aspecto problemático, por la distancia de las pequeñas localidades analizadas con respecto a los principales centros de consumo y la ausencia de canales de venta establecidos, en especial en el caso de las producciones no tradicionales (como la cría de conejos). En los productos de huerta, el desarrollo de mercados basados en la proximidad presenta complejidades particulares en áreas como las pampeanas caracterizadas por la producción de commodities, y donde el productor por lo general no suele involucrarse en la comercialización. 
Varias de estas cuestiones son percibidas por los funcionarios nacionales que se desempeñan en áreas de apoyo a microemprendimientos:

Mi pensamiento es ¿por qué no hay gente nueva? Porque todo emprendimiento primario lleva un proceso, vos desde que pones la semilla y te da el primer producto para poder salir a vender, mientras tanto ¿qué hace el emprendedor? [...] Comúnmente los emprendedores tienen 50 colmenas, dos vaquitas y tienen la leche, son emprendimientos que tienen varias actividades a la vez, por el tema de la estacionalidad. [...] Van armando y complementando para armar un flujo. Entonces la actividad agropecuaria de cero es difícil. [...] Además por el conocimiento, además de la apoyatura tenés que tener cierta preparación. Pero me apoyo en el proceso de espera hasta generar el primer ingreso monetario, porque la mayoría se larga a hacer el emprendimiento por necesidad. Necesitan mantener una familia, comer ellos, lo que sea, necesitan ya generar algo (técnico del Instituto Nacional de Tecnología Industrial, 2007).

La otra cuestión es conseguir la tierra [...] me imagino que con la rentabilidad del sector deben estar bastante caras. [...] Yo no sé cuál es la inversión por puesto de trabajo en el sector agropecuario comparado con otro sector de la economía, pero me parece que eso es un dato muy importante, y las capacitaciones requeridas para el sector agropecuario, comparadas con las que se requieren para otras actividades [...] Yo me imagino que en este momento entrar en el sector agropecuario, no es que no haya demanda, sino que te tenés que meter en un campo minado. [...] Están mucho más expuestos que hace 10 años a los cambios climáticos los productores agropecuarios y el [productor] chico tiene menos margen de soportarlo (técnica del Ministerio de Desarrollo Social, Programa "Manos a la Obra", 2007).

La viabilidad de esto tiene que ver con que a medida que esto se vaya afianzando, tiene que estar acompañado de una política y hay que construir el encuadre legal y reglamentario de viabilidad, hoy en día no está favorecida. Toda la legislación y la habilitación bromatológica están pensadas para el empresario (funcionario del Instituto Nacional de Tecnología Agropecuaria, Programa PROHUERTA).

Algunas de las restricciones aludidas hacen al contexto y al tipo de políticas aplicadas. Ahora bien, ¿qué hicieron en los casos estudiados para enfrentar estos problemas? En el cuadro siguiente se mencionan algunos de los mecanismos empleados.

Se destaca el papel clave de las ayudas logradas mediante lazos sociales diádicos (y complementariamente, la debilidad de estrategias asociativas más abarcadoras), la importancia de los apoyos institucionales, el lugar central de las restantes ocupaciones, en tanto permiten obtener los ingresos necesarios para la reproducción familiar y para hacer frente a gastos demandados por el emprendimiento. El trabajo asalariado del microemprendedor en la misma producción en la que trabaja de forma autónoma a veces le posibilita el acceso a herramientas, como forma de pago no monetaria (Murmis y Feldman, 2003). Sin embargo, es una modalidad encontrada en la apicultura pero no necesariamente en otras actividades iniciadas con un capital reducido. 
Cuadro 6

Estrategias desplegadas por los sujetos para enfrentar las barreras de acceso a la actividad agropecuaria

\begin{tabular}{|c|c|c|}
\hline Barreras & Estrategias individuales/diádicas & $\begin{array}{c}\text { Estrategias grupales } \\
\text { o con apoyos institucionales }\end{array}$ \\
\hline $\begin{array}{l}\text { Acceso a la tierra/ } \\
\text { Instalaciones }\end{array}$ & $\begin{array}{l}\text { Préstamo de tierra de conocidos (a veces } \\
\text { a cambio de pequeñas contribuciones a la } \\
\text { cosecha). } \\
\text { Empleo de terreno/galpón disponible en la } \\
\text { vivienda familiar. } \\
\text { Préstamo de instalaciones de procesamiento } \\
\text { por parte de familiares o productores más } \\
\text { consolidados. }\end{array}$ & $\begin{array}{l}\text { Tierra y galpón cedidos por escuelas agrarias } \\
\text { Apoyo proveniente de programas nacionales para } \\
\text { la construcción de instalaciones colectivas de } \\
\text { procesamiento. }\end{array}$ \\
\hline $\begin{array}{l}\text { Acceso a herramientas, } \\
\text { maquinaria }\end{array}$ & $\begin{array}{l}\text { Aportes provenientes del trabajo asalariado } 0 \\
\text { de ahorros previos. } \\
\text { Armado de instalaciones por parte del } \\
\text { emprendedor para abaratar costos. } \\
\text { Herramientas obtenidas a cambio de trabajo } \\
\text { con productores más consolidados. } \\
\text { Crédito en condiciones blandas (si bien en } \\
\text { escasa medida). }\end{array}$ & $\begin{array}{l}\text { Apoyo proveniente de programas nacionales o de } \\
\text { grandes empresas que realizan acciones de RSE.* }\end{array}$ \\
\hline $\begin{array}{l}\text { Acceso al capital } \\
\text { circulante }\end{array}$ & $\begin{array}{l}\text { Aportes provenientes del trabajo asalariado. } \\
\text { Traslados a cambio de trabajo/ ayudas de } \\
\text { conocidos y familiares. } \\
\text { Donaciones de conocidos, empleados para } \\
\text { preparar alimentos balanceados. } \\
\text { Pago de insumos al momento de la venta de } \\
\text { la producción. } \\
\text { Reducción de gastos monetarios. }\end{array}$ & $\begin{array}{l}\text { Apoyo de programas nacionales materializado en } \\
\text { insumos iniciales. } \\
\text { Gastos corrientes (electricidad) a cargo de escuelas } \\
\text { agrarias. Adelantos de dinero para insumos, que se } \\
\text { reponen con la venta de la producción, por parte de } \\
\text { instituciones estatales locales. } \\
\text { Préstamo de camiones municipales para el reparto } \\
\text { de la producción. } \\
\text { Gastos de traslado compartidos entre varios } \\
\text { productores. } \\
\text { Compra de insumos en conjunto para abaratar costos } \\
\text { (en menor medida). }\end{array}$ \\
\hline $\begin{array}{l}\text { Acceso a mano de obra } \\
\text { para cosecha y otras } \\
\text { tareas demandantes de } \\
\text { trabajo }\end{array}$ & $\begin{array}{l}\text { Ayuda de conocidos } \\
\text { Intercambios de trabajo }\end{array}$ & \\
\hline Acceso al mercado & $\begin{array}{l}\text { Venta por medio de terceros (familiares) } \\
\text { inscriptos. } \\
\text { Venta por medio de terceros ya consolidados, } \\
\text { con mayor producción. } \\
\text { Venta en mercados locales (comercios o } \\
\text { clientes particulares), sin facturar. }\end{array}$ & $\begin{array}{l}\text { Difusión de los emprendimientos y contactos con } \\
\text { clientes posibilitados por el municipio. } \\
\text { Compra de parte de la producción por comedores y } \\
\text { hospitales municipales. } \\
\text { Uso de facturas y números de inscripción } \\
\text { "institucionales". } \\
\text { Tolerancia municipal frente a requisitos } \\
\text { bromatológicos de las instalaciones. }\end{array}$ \\
\hline $\begin{array}{l}\text { Demora en obtención de } \\
\text { ingresos/ Estacionalidad }\end{array}$ & $\begin{array}{l}\text { Compensados con ingresos provenientes del } \\
\text { trabajo asalariado. }\end{array}$ & $\begin{array}{l}\text { Constitución de un "fondo de reserva", para } \\
\text { compensar momentos de producción escasa o nula, } \\
\text { complementado con subsidios municipales. }\end{array}$ \\
\hline
\end{tabular}

Fuente: Elaboración propia con base en entrevistas.

* Responsabilidad Social Empresarial. 


\section{Algunos factores contextuales que inciden en la viabilidad de estos emprendimientos}

En el apartado anterior se mencionó la presencia de antecedentes personales o familiares en la actividad agraria en varios de los casos estudiados que posibilitaron el acceso a saberes importantes para el desarrollo de la actividad. El análisis de las entrevistas muestra también el hecho de que en la zona hayan antecedentes en los rubros productivos elegidos es fundamental, porque implica "masa crítica", supone la presencia de servicios conexos y de referentes técnicos; también se asocia con la existencia de espacios de sociabilidad en donde se intercambian conocimientos y se resuelven problemáticas comunes. Si bien en algunos rubros productivos la multiplicación de microemprendimientos puede implicar competencia por recursos escasos (por ejemplo, en relación con los campos donde ubicar las colmenas), es necesario un "piso" mínimo de productores para estar en la posibilidad de sustentar procesos incipientes de desarrollo local.

Aun así, la inserción dominante de la región pampeana con base en la producción de commodities, con tendencia al incremento de las escalas operativas, hace que el espectro de antecedentes en materia de actividades encaradas con bajo capital sea limitado. La ausencia de las "externalidades" arriba mencionadas se nota en el caso de la cría de conejos, actividad fomentada por el gobierno provincial, y donde se carecía de técnicos capacitados en el ámbito local que pudieran acompañar a los emprendimientos. Pero también se da en actividades menos "novedosas" como la horticultura; es frecuente que las pequeñas localidades sean provistas de frutas y verduras por mercados distantes.

En contraste, la producción de miel es una actividad conocida en la provincia de Buenos Aires; tiene significación desde el punto de vista económico y, al mismo tiempo, puede ser considerada como un caso exitoso de inserción de pequeños y medianos productores, en virtud de la importante participación que tradicionalmente éstos han tenido en la actividad. ${ }^{6}$

Los procesos más generales que se examinaron en el segundo apartado — sobre todo la agriculturización — han ido sin embargo acotando el margen para su desarro-

6 Según datos de la Secretaría de Agricultura, Ganadería, Pesca y Alimentos (SAGPYA), Argentina es el primer exportador mundial de miel a granel, con 98,800 toneladas en 2007. En la actualidad, la actividad apícola es desarrollada por más de 30,000 productores, de los cuales $23 \%$ se encuentra en la provincia de Buenos Aires, que concentra más de $40 \%$ de la producción nacional. Se conjetura que al menos $75 \%$ del total de los apicultores en el ámbito nacional cuenta con menos de las 350 colmenas necesarias para vivir de la actividad (Estrada, 2006). 
1lo. Diferentes trabajos recientes aluden a la merma de rendimientos y a la necesidad de los apicultores de desplazarse hacia zonas más lejanas. Los cereales y oleaginosos desarrollados en la región no generan flora melífera —excepto el girasol, pero éste no alcanza $10 \%$ de la superficie implantada (véase cuadro 2) - . A ello se suma la diferente forma de llevar a cabo los trabajos: la aplicación de una nueva generación de herbicidas asociados a la agricultura (más potentes, con acción residual) y el manejo más intensivo de la ganadería — donde las pasturas no se dejan florecer-erradicaron gran parte de la flora natural de los campos así como la existente en las banquinas de los caminos (Estrada, 2006).

La confluencia de este conjunto de factores ha llevado a una disminución de apicultores en los distritos ubicados sobre la zona núcleo cerealera (la región agrícolaganadera del norte), concentrándose en el sector oeste, suroeste y sureste de Buenos Aires (Musicco, 2006). Sin embargo, aún en estas últimas se verifica la disminución de los rendimientos y la necesidad de recurrir al traslado de colmenas hacia otras provincias, como La Pampa y Río Negro. Esta práctica, antes inexistente, incrementa los costos de los apicultores; en caso de permanecer en el lugar, deben emplear azúcar para alimentarlas. Otros factores contextuales también tienden al desplazamiento de los apicultores más pequeños, como es el caso del "endurecimiento" de la normativa destinada a asegurar la trazabilidad de la miel, a partir de las exigencias establecidas por los mercados externos. ${ }^{7}$

Y no sólo desaparecieron las pasturas, sino que desapareció el sistema de manejo de las pasturas con respecto a cómo se consume esa pastura, que antes se dejaba entrar en floración y recién se la consumía; hoy se está consumiendo en una etapa más adelantada. [...] Incluso hay muchos apicultores que están pensando, en alguna época del año, trasladar las colmenas a algún otro lugar, que antes a lo mejor no se hacía [...] Qué se yo [...] irnos a La Pampa [...] Incluso por el control de malezas, que antes la abeja aprovechaba mucho las malezas: cardos, mostacillas... y ahora con el control de las malezas, por una cuestión obvia, casi ha dejado de haber malezas. [...] Creo que se quejan mucho, sí, sobre todo porque la soja les quitó girasol, las malezas que antes usaban y la floración de las pasturas, que no llegan a floración (miembro de la Comisión Directiva de Sociedad Rural, 2007).

De lo que yo he visto estos últimos años, [la apicultura] está en su peor parte. Te doy un ejemplo: yo, por ejemplo, ahora estaba dando un kilo por semana por colmena de alimento, porque estoy terminando un medicamento que me sale cinco pesos por colmena, pero termino y empiezo a dar tres kilos por semana, porque no les está alcanzando, no se están

7 Entre ellas se cuenta la obligación de estar inscrito en el registro de productores apícolas (RENAPA) y de realizar la extracción en salas registradas. En el cuadro 6 se mencionaron algunas de las estrategias desplegadas por los productores para hacer frente a estas exigencias. 
desarrollando porque no les alcanza la comida. Cuando en este momento las colmenas se tendrían que estar enjambrando solas (técnico apícola, 2007).

Al no haber flor no podés [...] hacer maravillas [...] Tenés que darles de comer azúcar [...] mucha azúcar [...] Cuando antes yo tenía 40 colmenas vivían y les dejaba lo que sobraba [de miel] para que ellas pasen el invierno. Y era ideal, porque tenías menos trabajo, las abejas estaban más sanas (apicultor, 2007).

Estos rasgos conllevan la necesidad de políticas que permitan incidir no ya en el nivel micro de los emprendimientos, sino en el nivel "meso", aquel vinculado a las regulaciones, las articulaciones con otros actores, los mecanismos de comercialización, y el acceso a recursos críticos. Por ello, desde los ámbitos locales se visualiza la necesidad de actuar sobre estos aspectos, así como en otros vinculados a las actividades que estructuran las economías de los distritos.

\section{A modo de conclusión}

En la provincia de Buenos Aires, como en gran parte de la región pampeana argentina, la disminución de la población ocupada en actividades agrarias se vincula con la concentración de la producción en un menor número de establecimientos, la profundización del proceso de agriculturización sustentado en la producción de cereales y oleaginosas, y la intensificación productiva, a partir de cambios tecnológicos ahorradores de mano de obra. En este sentido, si bien la expansión de la soja como "producto estrella" ha generado significativas consecuencias en áreas no pampeanas, no es menos cierto que también ha producido cambios en zonas de la región pampeana antes incorporadas marginalmente a esta producción, a partir del desplazamiento o disminución de actividades que compiten por el recurso del suelo, como la ganadería de invernada y el tambo. Esta situación se ha visto acentuada en el periodo iniciado con posterioridad a 2002, en que se modificó la política macroeconómica argentina, generando el reposicionamiento de las actividades orientadas a la exportación en un contexto internacional signado por una mayor demanda de materias primas.

A partir del estudio de estas condiciones generales, que han incidido en la consolidación de bolsones de desempleo en áreas rurales y pequeñas localidades pampeanas profundamente imbricadas con la actividad agropecuaria, el trabajo analizó las modalidades que asumen las actividades de autoempleo agropecuario de sujetos vulnerables, encaradas a partir de 2000. Se evaluó si las trayectorias ocupacionales de éstos presentaban vinculaciones previas con actividades autónomas y con el sector agropecuario, y se consideraron sus características como productores, identificándose 
algunas facetas que daban cuenta de limitaciones para que tales actividades se constituyeran en un mecanismo importante de reproducción material de sus hogares.

Claramente algunas de ellas exceden el nivel "micro" de los emprendimientos. Los procesos de amplio alcance que están influyendo en las áreas estudiadas inciden de manera significativa en la sustentabilidad y replicabilidad de las iniciativas emprendidas y permiten plantear la interrogante en la medida en que los factores que afectaron a estos sujetos —en tanto trabajadores asalariados — no vuelven a manifestarse, ya desde otro lugar, en su desarrollo como trabajadores autónomos.

No obstante, modificar su direccionalidad supone una gran apuesta por generar o reforzar procesos de desarrollo local en situaciones donde las dinámicas territoriales parecen condicionadas por reglas de juego y actores cada vez menos localizados, como es el caso de los pools de siembra. El apoyo a microemprendimientos asume como válidas ciertas premisas que deben discutirse a la luz de las tendencias identificadas. En otras palabras, esta investigación de nuevo pone en primer plano la necesaria articulación entre los niveles micro, meso y macro, mediante políticas que van más allá de pequeños apoyos a iniciativas aisladas, y reviertan no sólo los procesos de exclusión de productores preexistentes, sino también a viabilizar la inclusión de quienes consideran la actividad como mecanismo de sustento pra sus familias.

Por otra parte, las dificultades enfrentadas para iniciar y sostener actividades productivas agrarias en situaciones caracterizadas por el soporte fundamental del trabajo familiar nos suministran ciertas pistas acerca de la dinámica de cambio en un contexto globalizado de la estructura agraria de la provincia de Buenos Aires, como representativa de la región pampeana, al poner en evidencia procesos de expulsión de pequeños productores que se combinan con canales reducidos para posibilitar el ingreso "desde abajo", como "cuentapropistas" con un pequeño capital. La capacidad de desarrollar estrategias que desde el plano local pongan en cuestión las tendencias mencionadas, no sólo en el plano estrictamente productivo -mediante actividades alternativas y más intensivas en trabajo-, sino también en el plano de lo asociativo-organizativo, constituye una interrogante para explorar en futuras investigaciones. 


\section{ClARA VIRGINIA CRAVIOTTI}

Bibliografía

Banco Mundial, Argentina. Agricultura y Desarrollo rural: Temas claves, Informe 32763AR, Buenos Aires, junio de 2006.

Barsky, Andrés, "La puesta en valor y producción del territorio como generadora de nuevas geografías. Propuesta metodológica de zonificación agroproductiva de la Pampa argentina a partir de los datos del Censo Nacional Agropecuario 1988", en Osvaldo Barsky y Alfredo Pucciarelli (eds.), El agro pampeano. El fin de un periodo, Buenos Aires, FLACSO-Universidad de Buenos Aires, 1997, pp. 407-522.

Barsky, Osvaldo y Luciano Fernández, Tendencias actuales de las economías extrapampeanas, con especial referencia a la situación del empleo rural, RIMISP, documento elaborado en el marco de la preparación de la Estrategia Nacional de Desarrollo Rural para la Argentina, Buenos Aires, 2005.

Benencia, Roberto y Germán Quaranta, "Los mercados de trabajo agrarios en la Argentina: demanda y oferta en distintos contextos históricos", en Estudios del Trabajo, núm. 32, 2007, pp. 81-119.

Chudnovsky, Daniel, Sebastián Rubin, Eugenio Cap y Eduardo Trigo, Comercio internacional y desarrollo sustentable, Documento de trabajo núm. 25, Buenos Aires, Centro de Investigaciones para la Transformación, 1999.

Estrada, María Emilia, "El complejo apícola en el sudoeste bonaerense. Perfil de los agentes y formas de coordinación, en S. Gorenstein y V. Viego (comps.), Complejos productivos basados en recursos naturales y desarrollo territorial. Estudios de caso en Argentina, Bahía Blanca, Ediciones Universidad Nacional del Sur, 2006, pp. 105-128.

Gorenstein, Silvia, "Rasgos territoriales en los cambios del sistema agroalimentario pampeano (Argentina)", en EURE. Revista Latinoamericana de Estudios Urbanos Regionales, vol. 27, núm. 78, 2000.

Gutman, Graciela y Silvia Gorenstein, "Territorio y sistemas agroalimentarios, enfoques conceptuales, dinámicas recientes en Argentina", Desarrollo Económico, vol. 43, núm. 168, Buenos Aires, 2003, pp. 563-587.

Lattuada, Mario, "El crecimiento económico y el desarrollo sustentable en los pequeños y medianos productores agropecuarios argentinos de fines del Siglo XX", Taller Políticas públicas, institucionalidad y desarrollo rural en América Latina, FAOGobierno de México, México, 28-30 de agosto de 2000.

Lomnitz, Larissa Adler de, Cómo sobreviven los marginados, México, Siglo XXI Editores, 1975.

Murmis, Miguel y Silvio Feldman, "Persistencia de la pequeña producción mercantil en un pueblo rural: factores favorables y factores limitantes, ¿situación excepcional o situación generalizable?, en Revista Interdisciplinaria de Estudios Agrarios, núm. 19, segundo semestre, 2003, pp. 73-94.

Mussico, Miguel, Relevamiento y actualización de datos del registro provincial de marcas apícolas y de establecimientos procesadores de productos apícolas, Ministerio de Asuntos Agrarios de la provincia de Buenos Aires, Dirección Provincial de Desarrollo Rural, Departamento Animales Menores de Granja, 2006.

Neiman, Guillermo, Silvia Bardomás, Matías Berger, Mariela Blanco, Dora Jiménez y Germán Quaranta, Los asalariados del campo en la Argentina. Diagnóstico y politicas, Buenos Aires, PROINDER-DDA, Serie Estudios e Investigaciones, núm. 9.

Pizarro, Roberto, La vulnerabilidad social y sus desafíos: una mirada desde América Latina, CEPAL, Serie Estudios Estadísticos y Prospectivos, núm. 6, 2001.

Rofman, Alejandro, Desarrollo regional y exclusión social. Transformaciones y crisis en la Argentina contemporánea, Buenos Aires, Amorrortu Editores, 2000.

Saraví, Gonzalo, "Nuevas realidades y nuevos enfoques: Exclusión social en América Latina”, en Gonzalo Saraví (ed.), De la pobreza a la exclusión. Continuidades y rupturas de la cuestión social en América Latina, Buenos Aires, Prometeo, 2006, pp. 19-52.

Tort, María Isabel y Marcela Román, "Explotaciones familiares: diversidad de conceptos y criterios operativos", en María del Carmen González (coord.), Productores familiares pampeanos: Hacia la comprensión de similitudes y diferenciaciones zonales, Buenos Aires, Editorial Astralib, 2005, pp. 35-65. 